\title{
NONLINEAR MAPPINGS IN LOCALLY CONVEX SPACES $\left({ }^{1}\right)$
}

\author{
BY
}

TERRENCE S. McDERMOTT

\begin{abstract}
A notion of local linear approximation is defined for a nonlinear mapping, $f$, defined on one locally convex linear topological space with values in another. By use of this notion, theorems on the local solvability of the equation $y=f(x)$ and on the existence of a local inverse for $f$ are obtained. The continuity and linear approximability of the inverse are discussed. In addition, a relationship between the notion of linear approximation used in the paper and the notion of Fréchet differentiability is shown in the case the intervening spaces are Banach spaces.
\end{abstract}

In the ensuing discussion, we shall develop a notion of local linear approximation for nonlinear mappings between locally convex linear topological spaces. On the basis of this notion, we shall obtain several mapping theorems, the fundamental one being Theorem 3. If $f$ is a nonlinear mapping defined on an open subset, $N$, of a locally convex space $E$ with values in a locally convex space $F$, and if, for convenience, $0 \in N$ and $f(0)=0$, this theorem gives conditions for there to exist a neighborhood $U$ of 0 in $E$ such that $f(U)$ contains a neighborhood of 0 in $F$. Our results are related to those of L. M. Graves [4] and R. G. Bartle [2], the context of the discussion of Graves being Banach spaces, that of Bartle's work being metric groups. The line of thought followed in the present article will be pursued in a subsequent paper, where the problem of implicit functions will be taken up. At that time, we will give an application to the problem of global existence and uniqueness of solutions for a class of nonlinear Volterra integral equations.

Throughout this article, the letters $E$ and $F$ will denote Hausdorff real locally convex linear topological spaces, $N$ an open subset of $E$ and $f$ a mapping from $N$ into $F$. If $A$ is any absolutely convex (i.e. convex and balanced) set in $E$, the seminorm associated with $A$, defined on the subspace $E_{A}$ of $E$ generated by $A$, will be denoted by $\|\cdot\|_{A}$. That is, we have $\|x\|_{A}=\inf \{\lambda>0: x \in \lambda A\}$ for all $x \in E_{A}$. If $A$ is bounded, $\|\cdot\|_{A}$ is a norm on $E_{A}$. In particular, $\|x\|_{A}=0$ implies $x=0$ in this

Received by the editors July 11, 1969 and, in revised form, February 10, 1970.

AMS 1970 subject classifications. Primary 47H15; Secondary 46A05, 58C15.

Key words and phrases. Bounded set, local linear approximation, open mapping, continuous inverse.

( $\left.{ }^{1}\right)$ The results in this article are essentially contained in the author's doctoral dissertation completed under the guidance of Dr. Donald H. Hyers at the University of Southern California, 1969. 
case. If $\alpha>0, E_{\alpha A}$ and $E_{A}$ are identical, and the relation

$$
\|x\|_{\alpha A}=\frac{1}{\alpha}\|x\|_{A}
$$

holds for all $x \in E_{A}$. It is easy to verify that if $U$ is any absolutely convex neighborhood of 0 in $E$, then $E_{A \cap U}=E_{A}$.

Definition 1. A class $\mathscr{B}$ of subsets of $E$ shall be called a bounded covering for $E$ if the following three conditions hold:

(C1) Each $B \in \mathscr{B}$ is bounded and absolutely convex.

(C2) If $B \in \mathscr{B}$, then $\lambda B \in \mathscr{B}$ for all real $\lambda \geqq 1$.

(C3) $\bigcup_{B \in \mathscr{B}} B=E$.

It is a simple matter to construct examples of bounded coverings for many familiar spaces.

EXAmple 1. Let $E$ be a normed linear space, $B_{0}$ the closed unit ball in $E$. $\mathscr{B}=$ $\left\{\lambda B_{0}: \lambda>0\right\}$ is a bounded covering for $E$.

ExAmple 2. Let $E$ be arbitrary. For each $x \in E$, define $B_{x}=\{y \in E: y=\alpha x$, $|\alpha| \leqq 1\} . \mathscr{B}=\left\{B_{x}: x \in E\right\}$ is a bounded covering for $E$.

EXAMPLE 3. Let $E$ be the strict inductive limit of the sequence of real normed spaces $E_{1} \subset E_{2} \subset E_{3} \subset \ldots$. If $B_{n}$ is the closed unit ball in $E_{n}$, then $\mathscr{B}=\left\{\lambda B_{n}: \lambda>0\right.$, $n=1,2, \ldots\}$ is a bounded covering for $E$.

Definition 2. Let $\mathscr{B}$ be a bounded covering for $E$, and let $x_{0}$ be an element of $N$. Suppose $U$ is an absolutely convex neighborhood of 0 in $E$ and $\delta>0$ is a given real number. A linear mapping $L: E \rightarrow F$ will be said to $(\delta, \mathscr{B})$-approximate $f$ at $x_{0}$ on $U$ if the following four conditions hold:

(A1) $x_{0}+U \subset N$.

(A2) $L(B)$ is bounded in $F$ for every $B \in \mathscr{B}$.

(A3) $(f-L)\left(x_{0}+(B \cap U)\right) \subset(f-L) x_{0}+F_{L(B \cap U)}$ for all $B \in \mathscr{B}$.

(A4) For each $B \in \mathscr{B},\left\|(f-L) x_{1}-(f-L) x_{2}\right\|_{L(B \cap U)} \leqq \delta\left\|x_{1}-x_{2}\right\|_{B \cap U}$ for all $x_{1}$ and $x_{2}$ in $x_{0}+(B \cap U)$.

We remark that condition (A3) is required primarily to ensure that the left-hand side of the inequality in (A4) is defined. Our first result serves to relate this notion of local linear approximation to that of Fréchet differentiability in the case that the intervening spaces are Banach spaces.

Theorem 1. Let $E$ and $F$ be Banach spaces, $x_{0} \in N$. Suppose $f$ is continuously Fréchet differentiable on $N$, and that $f^{\prime}\left(x_{0}\right)$ is onto $F$. Then, given any $\delta>0$ and any neighborhood $V$ of 0 in $E$, there exists a neighborhood $U$ of 0 contained in $V$ on which $f^{\prime}\left(x_{0}\right)(\delta, \mathscr{B})$-approximates $f$ at $x_{0}$, where $\mathscr{B}$ is the bounded covering of Example 1.

Proof. Denote $f^{\prime}\left(x_{0}\right)$ by $L$. Since $L$ is continuous and onto $F$, it is an open mapping. Consequently, there exists $\alpha>0$ with $L\left(\alpha B_{0}\right) \supset A_{0}=\{y \in F:\|y\| \leqq 1\}$. Let $\delta^{\prime}=\delta / \alpha$. Since $f$ is continuously differentiable, we have (see (8.6.2) of [3]) that $\left\|f\left(x_{1}\right)-f\left(x_{2}\right)-L\left(x_{1}-x_{2}\right)\right\| \leqq \delta^{\prime}\left\|x_{1}-x_{2}\right\|$ for all $x_{1}$ and $x_{2}$ in a sufficiently small 
ball, say $x_{0}+\beta B_{0}$, about $x_{0}$. In Definition 2 , let $U=\beta B_{0}$. It is clear then that (A1), (A2), and (A3) are satisfied. To obtain (A4), we write

$$
\begin{aligned}
(1 / \alpha)\left\|f\left(x_{1}\right)-f\left(x_{2}\right)-L\left(x_{1}-x_{2}\right)\right\|_{L\left(B_{0}\right)} & =\left\|f\left(x_{1}\right)-f\left(x_{2}\right)-L\left(x_{1}-x_{2}\right)\right\|_{L\left(\alpha B_{0}\right)} \\
& \leqq\left\|f\left(x_{1}\right)-f\left(x_{2}\right)-L\left(x_{1}-x_{2}\right)\right\|_{A_{0}} \\
& =\left\|f\left(x_{1}\right)-f\left(x_{2}\right)-L\left(x_{1}-x_{2}\right)\right\| \\
& \leqq \delta^{\prime}\left\|x_{1}-x_{2}\right\|_{B_{0}} .
\end{aligned}
$$

Consequently,

$$
\left\|f\left(x_{1}\right)-f\left(x_{2}\right)-L\left(x_{1}-x_{2}\right)\right\|_{L\left(B_{0}\right)} \leqq \alpha \delta^{\prime}\left\|x_{1}-x_{2}\right\|_{B_{0}}=\delta\left\|x_{1}-x_{2}\right\|_{B_{0}} .
$$

Given any $B=\lambda B_{0} \in \mathscr{B}$, we have $U \cap B=\lambda B_{0}$ or $\beta B_{0}$. But for any real $h>0$, we obtain from the last inequality that

$$
\left\|f\left(x_{1}\right)-f\left(x_{2}\right)-L\left(x_{1}-x_{2}\right)\right\|_{L\left(n B_{0}\right)} \leqq \delta\left\|x_{1}-x_{2}\right\|_{h B_{0}} .
$$

Hence, (A4) is satisfied.

We now proceed to obtain a useful characterization of the injective property for a linear map $L: E \rightarrow F$.

THEOREM 2. A linear map $L: E \rightarrow F$ is injective if and only if there exists a positive number $m>0$ such that for every absolutely convex, bounded set $B \subset E$,

$$
\left\|L\left(x_{1}-x_{2}\right)\right\|_{L(B)} \geqq m\left\|x_{1}-x_{2}\right\|_{B}
$$

for all $x_{1}$ and $x_{2}$ in $B$. If $L$ is injective, then $m$ may be taken equal to 1 , and the inequality becomes an equality.

Proof. Suppose $L$ is injective. Let $B$ be any absolutely convex, bounded set in $E$, and suppose $x_{1}$ and $x_{2}$ are in $B$. Then

$$
\begin{aligned}
\left\|L\left(x_{1}-x_{2}\right)\right\|_{L(B)} & =\inf \left\{\lambda>0: L\left(x_{1}-x_{2}\right) \in \lambda L(B)\right\} \\
& =\inf \left\{\lambda>0: x_{1}-x_{2} \in \lambda B\right\}=\left\|x_{1}-x_{2}\right\|_{B},
\end{aligned}
$$

using the linearity of $L$. Taking $m=1$, we have (2). Now assume the condition of the theorem satisfied. Pick $x_{1}$ and $x_{2}$ in $E$, and suppose $L x_{1}=L x_{2}$. If $x_{1} \neq x_{2}$, let $B=\left\{\lambda\left(x_{1}-x_{2}\right):|\lambda| \leqq 1\right\}$. This set, $B$, is absolutely convex and bounded. We have, then, $0=\left\|L\left(x_{1}-x_{2}\right)\right\|_{L(B)} \geqq m\left\|x_{1}-x_{2}\right\|_{B}=m>0$, a contradiction. Hence, $x_{1}=x_{2}$, and therefore $L$ is injective.

We recall that if $M \subset E$ is a closed subspace of $E$, then $E / M$ is a Hausdorff, locally convex, linear topological space whose topology is comprised of all sets $W \subset E / M$ such that $\nu^{-1}(W)$ is open in $E$, where $\nu$ is the natural homomorphism of $E$ onto $E / M$. Since $\nu$ is then not only linear but continuous as well, a bounded covering $\mathscr{B}$ for $E$ is taken by $\nu$ into a bounded covering $\mathscr{B}^{\prime}=\{\nu(B): B \in \mathscr{B}\}$ for $E / M$. Given an absolutely convex, bounded set $B$ in $E$, our next result relates the norm $\|\cdot\|_{v(B)}$ defined on $(E / M)_{v(B)}$ to $\|\cdot\|_{B}$ defined on $E_{B}$. By abuse of notation, 
we shall use the same symbol, $\xi$, to denote a generic element of $E / M$, whether considered as an element of that vector space or as a subset of $E$.

In this setting, we have

LEMMA 1. For every $\xi \in(E / M)_{v(B)}$, we have the relation

$$
\|\xi\|_{v(B)}=\inf \left\{\|x\|_{B}: x \in \xi \cap E_{B}\right\},
$$

where $B$ is any absolutely convex, bounded set in $E$.

Proof.

$$
\begin{aligned}
\|\xi\|_{\nu(B)} & =\inf \{\lambda>0: \xi \in \lambda \nu(B)\} \\
& =\inf \{\lambda>0: \xi \in \nu(\lambda B)\} \\
& =\inf \{\lambda>0: \exists x \in \lambda B \text { with } \nu(x)=\xi\} \\
& =\inf \{\lambda>0: \lambda B \cap \xi \neq \varnothing\} .
\end{aligned}
$$

Let $K=\left\{\|x\|_{B}: x \in E_{B} \cap \xi\right\}$. Clearly $\|\xi\|_{v(B)} \geqq \inf K$. On the other hand, if $\|x\|_{B} \in K$, then for any real number $\gamma>\|x\|_{B}, x \in \gamma B \cap \xi$. Hence, all such $\gamma$ lie in $\{\lambda>0: \lambda B \cap \xi \neq \varnothing\}$, and thus $\|\xi\|_{\nu(B)} \leqq\|x\|_{B}$. But then, $\|\xi\|_{\nu(B)}=\inf K$.

We are now ready to prove a mapping theorem basic for our later work. The proof employs the notion of quotient space in a manner suggested by the proof (due to H. A. Antosiewicz [1]) of a similar result in the case the intervening spaces are Banach spaces.

THEOREM 3. Suppose $E$ is sequentially complete and that $\mathscr{B}$ is a bounded covering for $E$. Let $N$ be a neighborhood of the origin in $E$, and assume $f: N \rightarrow F$ is continuous on $N$ and that $f(0)=0$. If for some $\delta, 0<\delta<1$, there is an open linear map $L$ of $E$ onto $F$, having a closed null space, which $(\delta, \mathscr{B})$-approximates $f$ at $0 \in E$, then there exists an absolutely convex neighborhood $U$ of 0 in $N$ such that $f(U)$ contains an absolutely convex neighborhood $V$ of $0 \in F$. In fact, $V=L(((1-k) / 4) U)$, where $k=2 \delta /(1+\delta)$.

Proof. Denote the null space of $L$ by $M$. Since $M$ is closed, $E / M$ is a Hausdorff locally convex space as discussed above. We have the usual commuting diagram:

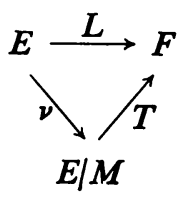

It is easily verified that, since $L$ is open and $\nu$ continuous, $T$ is open, as well as onto $F$. $T$, of course, is also injective. Let $U$ be a neighborhood of $0 \in E$ on which $L$ $(\delta, \mathscr{B})$-approximates $f$ at 0 , and define $k=2 \delta /(1+\delta)$. Set $V=L(((1-k) / 4) U)$. Since $L$ is linear and open, $V$ is an absolutely convex neighborhood of 0 in $F$. Let $y_{0}$ be 
an element of $V$, and set $\xi_{0}=0 \in E / M, x_{0}=0 \in E$. Define

$$
\xi_{1}=\xi_{0}-T^{-1}\left(f\left(x_{0}\right)-y_{0}\right) \text {. }
$$

Choose $x_{1}$ in $((1-k) / 4) U$ with $L x_{1}=y_{0}$, and find $B \in \mathscr{B}$ such that $x_{1} \in E_{B}$ and $\left\|x_{1}\right\|_{B \cap U} \leqq(1-k) / 4$. It follows that $\xi_{1}$ is in $(E / M)_{v(B \cap U)}$. We may assume without loss of generality that

$$
\left\|x_{1}-x_{0}\right\|_{B \cap U} \leqq(2-k)\left\|\xi_{1}-\xi_{0}\right\|_{v(B \cap U)} .
$$

This follows from Lemma 1 and the fact that $k<1$. Employing Theorem 2 in conjunction with the bijectivity of $T$, we have

$$
\begin{aligned}
\left\|\xi_{1}-\xi_{0}\right\|_{v(B \cap U)} & =\left\|T^{-1}\left(y_{0}\right)\right\|_{v(B \cap U)} \\
& =\left\|y_{0}\right\|_{T v(B \cap U)}=\left\|y_{0}\right\|_{L(B \cap U)} \leqq(1-k) / 4<(1-k) / 2,
\end{aligned}
$$

and, keeping in mind that $k<1$,

$$
\left\|x_{1}-x_{0}\right\|_{B \cap U} \leqq(2-k)\left\|\xi_{1}-\xi_{0}\right\|_{\nu(B \cap U)} \leqq(2-k)((1-k) / 4)<(1-k) / 2 .
$$

Now, suppose $x_{0}, x_{1}, \ldots, x_{n}$ and $\xi_{0}, \xi_{1}, \ldots, \xi_{n}$ have been found satisfying

(a,j) $\xi_{j}=\xi_{j-1}-T^{-1}\left(f\left(x_{j-1}\right)-y_{0}\right)$,

(b,j) $\nu\left(x_{j}\right)=\xi_{j}=T^{-1} L x_{j}$,

(c, $j)\left\|x_{j}-x_{j-1}\right\|_{B \cap U} \leqq(2-k)\left\|\xi_{j}-\xi_{j-1}\right\|_{v(B \cap U)}$,

$(\mathrm{d}, j)\left\|\xi_{j}-\xi_{j-1}\right\|_{v(B \cap U)} \leqq k^{j-1}\left\|\xi_{1}-\xi_{0}\right\|_{v(B \cap U)}$,

$(\mathrm{e}, j)\left\|x_{j}\right\|_{B \cap U}<\frac{1}{2}(1-k)\left(1+k+k^{2}+\cdots+k^{j-1}\right)<1$

for all $j=1,2, \ldots, n$.

We then define $\xi_{n+1}$ in accordance with $(\mathrm{a}, n+1)$ and obtain, using $(\mathrm{b}, n)$, $(\mathrm{b}, n-1)$ and $(\mathrm{a}, n)$,

$$
\xi_{n+1}-\xi_{n}=T^{-1}\left((L-f) x_{n}-(L-f) x_{n-1}\right) .
$$

But $x_{n}$ and $x_{n-1}$ are in $B \cap U$ by virtue of $(\mathrm{e}, n)$ and (e, $\left.n-1\right)$. The property (A3) of Definition 2, ensures that the right-hand side of equation (3) is in $(E / M)_{v(B \cap U)}$. Consequently, $\xi_{n+1} \in(E / M)_{v(B \cap U)}$. Using (3), Theorem 2 and condition (A4), we obtain from $(\mathrm{c}, n)$ that

$$
\begin{aligned}
\left\|\xi_{n+1}-\xi_{n}\right\|_{v(B \cap U)} & \leqq \delta\left\|x_{n}-x_{n-1}\right\|_{B \cap U} \\
& \leqq(k /(2-k))(2-k)\left\|\xi_{n}-\xi_{n-1}\right\|_{v(B \cap U)}=k\left\|\xi_{n}-\xi_{n-1}\right\|_{v(B \cap U)},
\end{aligned}
$$

which yields (d, $n+1)$. There exists $x_{n+1}$ satisfying both (b,n+1) and (c, $\left.n+1\right)$. Indeed, there exists

$$
x_{n+1}^{\prime} \in E_{B \cap U} \cap\left(\xi_{n+1}-\xi_{n}\right)
$$

with $\left\|x_{n+1}^{\prime}\right\|_{B \cap U} \leqq(2-k)\left\|\xi_{n+1}-\xi_{n}\right\|_{\nu(B \cap U)}$, according to Lemma 1 . Let $x_{n+1}=x_{n}+x_{n+1}^{\prime}$. Then $x_{n+1} \in \xi_{n+1}$, and $\left\|x_{n+1}-x_{n}\right\|_{B \cap U}=\left\|x_{n+1}^{\prime}\right\|_{B \cap U}$, which gives $(\mathrm{b}, n+1)$ and (c, $n+1)$. 
We establish $(\mathrm{e}, n+1)$ through the inequalities already established:

$$
\begin{aligned}
\left\|x_{n+1}\right\|_{B \cap U} & \leqq \sum_{j=1}^{n+1}\left\|x_{j}-x_{j-1}\right\|_{B \cap U} \\
& \leqq(2-k) \sum_{j=1}^{n+1}\left\|\xi_{j}-\xi_{j-1}\right\|_{v(B \cap U)} \\
& \leqq(2-k) \sum_{j=1}^{n+1} k^{j-1}\left\|\xi_{1}-\xi_{0}\right\|_{v(B \cap U)} \\
& \leqq(2-k)\left(\frac{1-k}{4}\right) \sum_{j=1}^{n+1} k^{j-1}<\frac{1-k}{2} \sum_{j=0}^{n} k^{j} .
\end{aligned}
$$

Thus, we have inductively defined the sequences $\left\{x_{n}\right\}$ and $\left\{\xi_{n}\right\}$ satisfying the properties $(\mathrm{a}, j)$ through $(\mathrm{e}, j)$ for all integers $j \geqq 1$. Using (c,j) and (d,j), it is easy to see that $\left\{x_{n}\right\}$ is a Cauchy sequence with respect to the norm $\|\cdot\|_{B \cap U}$. But the fact that $B \cap U$ is bounded in $E$ implies that $\left\{x_{n}\right\}$ is then also a Cauchy sequence with respect to the topology of $E$. Since $E$ is sequentially complete, $\left\{x_{n}\right\}$ converges to some point $x^{*}$ in $E$. $x^{*}$ may not be in $B$, but it must lie in $U$. In fact, this is seen from the inequalities

$$
\left\|x^{*}\right\|_{U} \leqq\left\|x^{*}-x_{n}\right\|_{U}+\left\|x_{n}\right\|_{U} \leqq\left\|x^{*}-x_{n}\right\|_{U}+\left\|x_{n}\right\|_{B \cap U}
$$

which hold for all $n \geqq 1$, by employing the inequalities $(\mathrm{e}, j)$ and the fact that $\left\{x_{n}\right\}$ converges in $E$ to $x^{*}$. That $f\left(x^{*}\right)=y_{0}$ follows by letting $n$ tend to infinity in

$$
\xi_{n+1}=\xi_{n}-T^{-1}\left(f\left(x_{n}\right)-y_{0}\right),
$$

keeping in mind that $\xi_{n}=\nu\left(x_{n}\right)$ converges to $\nu(x)$ and that $T^{-1}$ and $f$ are continuous.

Before continuing, we make the following observations:

REMARK 1. The assumption of continuity for $f$, while concise, is stronger than necessary. It suffices to ask only that $f$ be "sequentially continuous" in the sense that $x_{n} \rightarrow x$ implies $f\left(x_{n}\right) \rightarrow f(x)$ for all sequences $\left\{x_{n}\right\}$ in $N$ converging to a point in $N$.

REMARK 2. In the proof, we note for later reference, once the set $B \in \mathscr{B}$ is chosen such that $\left\|x_{1}\right\|_{B \cap U} \leqq(1-k) / 4$, the entire sequence $\left\{x_{n}\right\}$ lies in $B \cap U$, and its limit, $x^{*}$, will lie in $B$ if $B$ is closed under sequential limits.

COROLlaRY 3.1. The theorem remains true when in the hypotheses of the theorem the origin, 0 , in $E$ is replaced by an arbitrary element, $x_{0}$, of $E$. In this case, the conclusion takes the form: there exists an absolutely convex neighborhood $U$ of 0 in $E$ such that $x_{0}+U \subset N$, and $f\left(x_{0}+U\right) \supset f\left(x_{0}\right)+V$, where $V=L(((1-k) / 4) U)$, $k=2 \delta /(1+\delta)$. The assumption $f(0)=0$ should be omitted.

Proof. Apply the theorem to the map $g(x)=f\left(x_{0}+x\right)-f\left(x_{0}\right)$. The details are a straightforward verification that $g$ satisfies the hypotheses of the theorem. 
Definition 3. Let $\mathscr{B}$ be a bounded covering for $E$. If for each pair of points $x_{1}$ and $x_{2}$ in $E$ there exists a $B \in \mathscr{B}$ with both $x_{1} \in B$ and $x_{2} \in B$, then we say $\mathscr{B}$ is pair-containing.

The condition that a bounded covering be pair-containing is not particularly strong, though it is not always satisfied, as the covering in Example 2 shows in the extreme.

Definition 4. A subset $A$ of $E$ is said to be sequentially closed if, for each sequence $\left\{x_{n}\right\}$ contained in $A$ and convergent in $E$ to a point $x$ of $E$, the point $x$ is in $A$.

COROLlary 3.2. If, in addition to the hypotheses of Corollary 3.1, we assume that $\mathscr{B}$ is pair-containing and $L$ is injective, then for each $y \in f\left(x_{0}\right)+V$ there exists a unique $x \in x_{0}+U$ with $y=f(x), U$ and $V$ as in Corollary 3.1.

Proof. That at least one $x$ exists with $y=f(x)$ is a consequence of Corollary 3.1. Suppose $x_{1}$ and $x_{2}$ are in $U$ and $f\left(x_{0}+x_{1}\right)=f\left(x_{0}+x_{2}\right) \in f\left(x_{0}\right)+V$. Find $B \in \mathscr{B}$ with $x_{1} \in B$ and $x_{2} \in B$. We have then, using Theorem 2,

$$
\begin{aligned}
\delta\left\|x_{1}-x_{2}\right\|_{B \cap U} & \geqq\left\|f\left(x_{0}+x_{1}\right)-f\left(x_{0}+x_{2}\right)-L\left(x_{1}-x_{2}\right)\right\|_{L(B \cap U)} \\
& =\left\|L\left(x_{1}-x_{2}\right)\right\|_{L(B \cap U)}=\left\|x_{1}-x_{2}\right\|_{B \cap U} .
\end{aligned}
$$

But, since $\delta<1$, we have $\left\|x_{1}-x_{2}\right\|_{B \cap U}=0$. Since $B \cap U$ is bounded, this implies $x_{1}=x_{2}$.

Definition 5. We shall say $f: N \rightarrow F$ is smooth at $x \in N$ if there exists a base $\mathscr{N}_{x}$ of absolutely convex neighborhoods of 0 in $E$ such that for each $U \in \mathscr{N}_{x}$, there is a linear map $L_{U}: E \rightarrow F$, a bounded covering $\mathscr{B}_{U}$ for $E$ and a $\delta_{U}, 0<\delta_{U}<1$, such that $L_{U}\left(\delta_{U}, \mathscr{B}_{U}\right)$-approximates $f$ at $x$ on $U$. When $f$ is smooth at every $x \in N$, we shall say $f$ is smooth on $N$. If $f$ is smooth on $N$ and all the maps $L_{U}$ are open, onto $F$, and have closed null space, we shall say $f$ is strongly smooth on $N$.

THEOREM 4. Suppose $E$ is sequentially complete, and $f: N \rightarrow F$ is continuous. If $f$ is strongly smooth on $N$, then $f$ is an open map.

Proof. Let $K \subset N$ be an open set. Choose $x \in K$. The fact that $f$ is strongly smooth allows us to apply Corollary 3.1 to find an absolutely convex neighborhood $U$ with $x+U \subset K$ such that $f(x+U)$ covers a neighborhood of $f(x)$. But $f(x+U) \subset$ $f(K)$. Hence, $f(x)$ is interior to $f(K)$. Since $x$ was a general element of $K, f(K)$ is, therefore, open.

THEOREM 5. Suppose $E$ is sequentially complete, and $f: N \rightarrow F$ is continuous. Assume $f$ is strongly smooth on $N$, and (using the notation of Definition 5) that for some $x^{\prime} \in N$, there is a $U^{\prime}$ in $\mathscr{N}_{x^{\prime}}$ such that $L_{U^{\prime}}$ is injective and $\mathscr{B}_{U^{\prime}}$ is pair-containing. Then, there exists an absolutely convex neighborhood $U$ of 0 in $E$ such that $V=$ $f\left(x^{\prime}+U\right)$ is open in $F$ and $f$ has a continuous inverse defined on $V$ with values in $x^{\prime}+U$. 
Proof. Since $f$ is continuous, there is an absolutely convex neighborhood $U^{\prime \prime}$ of 0 in $E$ such that $f\left(x^{\prime}+U^{\prime \prime}\right) \subset f\left(x^{\prime}\right)+L_{U^{\prime}}\left(\left(1-k_{U^{\prime}}\right) U^{\prime} / 4\right)$, where $k_{U^{\prime}}=2 \delta_{U^{\prime}} /\left(1+\delta_{U^{\prime}}\right)$. Let $U=U^{\prime} \cap U^{\prime \prime}$. Then $V=f\left(x^{\prime}+U\right)$ is open (by Theorem 4) and is a subset of $f\left(x^{\prime}\right)+L_{U^{\prime}}\left(\left(1-k_{U^{\prime}}\right) U^{\prime} / 4\right)$. According to Corollary 3.2, we have that for each $y \in f\left(x^{\prime}+U\right)$, there is a unique $x \in x^{\prime}+U^{\prime}$ with $y=f(x)$. But, noting that $x^{\prime}+U \subset$ $x^{\prime}+U^{\prime}$, we see that, in fact, $x \in x^{\prime}+U$. Hence, there is an inverse mapping for $f$, defined on $V$ with values in $x^{\prime}+U$. Since $f$ is an open mapping on $N$ (and consequently on $x^{\prime}+U$ ), the inverse is continuous on $V$.

We remark that Corollary 3.2 implies the existence of a unique right inverse $g$ for $\left.f\right|_{x_{0}+U}$ defined on $f\left(x_{0}\right)+V$ with values in $x_{0}+U$. That is, $f(g(y))=y$ for all $y$ in $f\left(x_{0}\right)+V$, and $g(y) \in x_{0}+U$. The inverse function of Theorem 5 is just a restriction of the appropriate right inverse constructed from Corollary 3.2. In any case, the right inverse $g$ mentioned above is $(\beta, \mathscr{A})$-approximable at $f\left(x_{0}\right)$ for an appropriate choice of a bounded covering $\mathscr{A}$ for $F$ and of a number $\beta>0$. In this regard, we have

THEOREM 6. Let the hypotheses and notation of Corollary 3.2 be assumed. Then, there is a unique right inverse $g$ for $\left.f\right|_{x_{0}+U}$ mapping $f\left(x_{0}\right)+V$ into $x_{0}+U$. Furthermore, $L^{-1}: F \rightarrow E(\beta, \mathscr{A})$-approximates $g$ at $f\left(x_{0}\right)$ on $V$, where $\mathscr{A}=\{L(B): B \in \mathscr{B}\}$ and $\beta=\delta /(1-\delta)$, providing each $B \in \mathscr{B}$ is sequentially closed.

Proof. The existence of $g$ is clear, as remarked above. That $\mathscr{A}$ is a bounded covering for $F$ is a routine verification. Taking $N=f\left(x_{0}\right)+V$, the conditions (A1) and (A2) of Definition 2 are seen to be satisfied for $g$. For condition (A3), we need to show

$$
\left(g-L^{-1}\right)\left(f\left(x_{0}\right)+(A \cap V)\right) \subset\left(g-L^{-1}\right)\left(f\left(x_{0}\right)\right)+E_{L^{-1}(A \cap V)}
$$

for each $A \in \mathscr{A}$. Let $h(y)=g\left(y+f\left(x_{0}\right)\right)-g\left(f\left(x_{0}\right)\right)=g\left(y+f\left(x_{0}\right)\right)-x_{0}$. Then (4) is equivalent to

$$
\left(h-L^{-1}\right)(A \cap V) \subset E_{L^{-1}(A \cap V} .
$$

To show (5), we note $\left(h-L^{-1}\right)(A \cap V) \subset h(A \cap V)-L^{-1}(A \cap V)$. The only difficulty is in the first term on the right. Let $A \in \mathscr{A}$ and $A=L\left(B^{\prime}\right)$. Set $B=$ $(4 /(1-k)) B^{\prime}$. If $y \in A \cap V$, then $y \in L((1-k) B / 4 \cap(1-k) U / 4)$, using the fact that $L$ is injective. Hence, $L^{-1}(y) \in(1-k)(B \cap U) / 4$ and, consequently, $\left\|L^{-1}(y)\right\|_{B \cap U} \leqq$ $(1-k) / 4$. We now observe that $h(y)$ coincides with the unique right inverse, $t^{-1}$, mapping $V$ into $U$, for the map $t$ defined by $t(x)=f\left(x_{0}+x\right)-f\left(x_{0}\right)$. But following the construction of Theorem $3, t^{-1}(y)$ is the limit of the sequence $x_{0}=0, x_{n+1}(y)=$ $x_{n}(y)-L^{-1}\left(t\left(x_{n}(y)\right)-y\right)$. Thus $\left\|x_{1}(y)\right\|_{B \cap U}=\left\|L^{-1}(y)\right\|_{B \cap U} \leqq(1-k) / 4$. It follows from Remark 2 that $x=h(y) \in B \cap U$. Hence,

$$
\begin{aligned}
h(A \cap V) & \subset B \cap U \subset(4 /(1-k))(B \cap U) C \subset E_{B^{\prime} \cap U} \\
& =E_{B^{\prime}} \cap(1-k) U / 4=E_{L^{-1}(A \cap V) .}
\end{aligned}
$$

Thus, $h(A \cap V)-L^{-1}(A \cap V) \subset E_{L^{-1}(A \cap V)}$, and (5) is established. To obtain the 
inequality for $g$ corresponding to condition (A4), it suffices to show that for every $A \in \mathscr{A}$,

$$
\left\|h\left(y_{1}\right)-h\left(y_{2}\right)-L^{-1}\left(y_{1}-y_{2}\right)\right\|_{L^{-1}(A \cap V)} \leqq \beta\left\|y_{1}-y_{2}\right\|_{A \cap V}
$$

for all $y_{1}, y_{2}$ in $A \cap V$. From the remarks following (5), we see that $h\left(y_{1}\right)$ and $h\left(y_{2}\right)$ are in $B \cap U, B$ as above for a given $A$. Since $L(\delta, \mathscr{B})$-approximates $f$ at $x_{0}$ on $U$, we have, using the bijectivity of $L$ through Theorem 2 ,

$$
\begin{aligned}
\| y_{1}-y_{2}-L\left(h\left(y_{1}\right)\right. & \left.-h\left(y_{2}\right)\right) \|_{L(B \cap U)} \\
& \leqq \delta\left\|L\left(h\left(y_{1}\right)-h\left(y_{2}\right)\right)\right\|_{L(B \cap U)} \\
& \leqq \delta\left\|L\left(h\left(y_{1}\right)-h\left(y_{2}\right)\right)-\left(y_{1}-y_{2}\right)\right\|_{L(B \cap U)}+\delta\left\|y_{1}-y_{2}\right\|_{L(B \cap U)} .
\end{aligned}
$$

Hence,

$$
(1-\delta)\left\|L\left(h\left(y_{1}\right)-h\left(y_{2}\right)\right)-\left(y_{1}-y_{2}\right)\right\|_{L(B \cap U)}=\delta\left\|y_{1}-y_{2}\right\|_{L(B \cap U)}
$$

or using Theorem 2 again,

$$
\left\|h\left(y_{1}\right)-h\left(y_{2}\right)-L^{-1}\left(y_{1}-y_{2}\right)\right\|_{B \cap U} \leqq(\delta /(1-\delta))\left\|y_{1}-y_{2}\right\|_{L(B \cap U)} .
$$

But $B \cap U=(4 /(1-k)) L^{-1}(A \cap V)$, and hence

$$
\left\|h\left(y_{1}\right)-h\left(y_{2}\right)-L^{-1}\left(y_{1}-y_{2}\right)\right\|_{L^{-1}(A \cap V)} \leqq(\delta /(1-\delta))\left\|y_{1}-y_{2}\right\|_{A \cap V} .
$$

Since $\beta=\delta /(1-\delta)$, we are finished.

\section{REFERENCES}

1. H. A. Antosiewicz, Sufficient condition for controllability of nonlinear systems, Proc. Sem. Optimal Control and Hybr. Comput., CAS, Yugoslavia (to appear).

2. R. G. Bartle, Implicit functions and solutions of equations in groups, Math. Z. 62 (1965), 335-346. MR 17, 62.

3. J. Dieudonné, Foundations of modern analysis, Pure and Appl. Math., vol. 10, Academic Press, New York, 1960. MR 22 \#11074.

4. L. M. Graves, Some mapping theorems, Duke Math. J. 17 (1950), 111-114. MR 11, 729.

5. D. H. Hyers, Linear topological spaces, Bull. Amer. Math. Soc. 51 (1945), 1-21. MR 6, 275.

6. J. Sebastião e Silva, Le calcul différentiel et intégral dans les espaces localement convexes. I, Atti Accad. Naz. Lincei Rend. Cl. Sci. Fis. Mat. Natur. (8) 20 (1956), 743-750. MR 19, 561.

LOYOLA UNIVERSITY,

Los Angeles, California 90045 